Check for updates

Cite this: RSC Adv., 2019, 9, 33848

Received 25th August 2019

Accepted 16th October 2019

DOI: $10.1039 / c 9 r a 06688 \mathrm{~h}$

rsc.li/rsc-advances

\title{
Porous carbon from tobacco stalk for removal of organic dyes from water
}

\author{
Guo-Ning Guo, $\uparrow^{\mathrm{a}}$ Bin-Bin Yang, $\dagger^{\mathrm{b}}$ Qing-Mei Zhang ${ }^{\mathrm{b}}$ and Chun Zhang (D) *b
}

Tobacco stalk, a kind of agricultural residue, will cause environmental pollution because it contains some harmful substances such as nicotine. To realize the high-value utilization of this agricultural residue, we prepared porous carbon (TS-C) by high temperature carbonization using tobacco stalk as a precursor. It was found that TS-C displays a hierarchical pore structure and high Brunauer-Emmett-Teller (BET) surface area of $1416 \mathrm{~m}^{2} \mathrm{~g}^{-1}$. Moreover, TS-C has excellent performance in organic dye adsorption at room temperature, especially for Gentian violet (GV), with the maximum adsorption capacity of $926 \mathrm{mg} \mathrm{g}^{-1}$.

\section{Introduction}

Tobacco is one of the most important non-edible cash crops in the world. During the harvesting process of tobacco leaves, tobacco stalks, as agriculture residues, are collected in large amounts. ${ }^{1}$ As the largest country producing and consuming tobacco, there are more than 1.2 million tons of tobacco stalks produced in China every year. ${ }^{2}$ Discarding and incinerating tobacco stalk containing harmful substances such as nicotine, will cause air pollution and soil diseases. ${ }^{3}$ Therefore, how to use its potential resource value is one of the urgent problems that need to be solved in the tobacco industry.

Thus far, literature studies on the use of tobacco stalk mainly include the following aspects: (1) converting tobacco stalk into bio-oil by pyrolysis technology. ${ }^{4}$ But intensive energy is required for this process, and the quality of bio-oil will be affected by the pyrolysis of nicotine in tobacco stalk. (2) Using enzymes and microorganisms to convert tobacco stalk into sugar, lactic acid and other products by biorefinery technology. ${ }^{5}$ However, lignocellulose in tobacco stalk has complex physical structure and chemical connection, which will cause high resistance in the process of transformation, leading to low biotransformation efficiency. ${ }^{6}$ Because there are some deficiencies in the above methods, people still need to search for new ways of tobacco stalk resource utilization. Containing a large amount of cellulose and lignin, tobacco stalk can be used as precursor to prepare porous carbon materials. ${ }^{7}$

Porous carbon have played an important role in the fields of supercapacitors, lithium ion batteries, gas storage, photocatalysis, adsorption and separation with advantages of high

\footnotetext{
${ }^{a}$ Technology R\&D Center, Hubei Tobacco (Group) Co., Ltd., Wuhan, 430040, China ${ }^{b}$ College of Life Science and Technology, National Engineering Research Center for Nanomedicine, Huazhong University of Science and Technology, Wuhan, 430074, China.E-mail: chunzhang@hust.edu.cn

$\dagger$ These authors contributed equally to this work.
}

specific surface area, low density, diverse structure and high stability. ${ }^{8-10}$ However, most of the porous carbons are currently derived from fossil fuel derivatives or wood, and some of them are synthesized under harsh conditions or need to introduce toxic reagents, which are costly and harmful to the environment. ${ }^{11}$ Recently, we have synthesized porous carbon materials using hyper-crosslinked polymer ${ }^{12}$ as precursors for oxygen reduction reaction ${ }^{13}$ and iodine capture. ${ }^{14}$ By contrast, porous carbon materials prepared from biomass with a wide range of sources, have the advantages of low cost, large specific surface area and simple preparation, and resource utilization of biomass. ${ }^{15}$ In recent years, researchers have found that biomass porous carbon materials have broad potential application value in catalysis, adsorption, electrode materials and other fields. ${ }^{\mathbf{1 6 , 1 7}}$ Herein, a porous carbon (TS-C) with high specific surface area was prepared by high-temperature carbonization using agricultural residues tobacco stalk as precursor for removal of organic dyes from water.

\section{Results and discussion}

The tobacco stalk was pretreated at $500{ }^{\circ} \mathrm{C}$ for $2 \mathrm{~h}$ under nitrogen atmosphere, and then mixed with $\mathrm{KOH}$ at a mass ratio of $1: 4$, and treated at $700{ }^{\circ} \mathrm{C}$ for $2 \mathrm{~h}$ to obtain porous carbon.

The structure and morphology of TS-C were confirmed by Scanning electron microscope (SEM) and transmission electron microscope (TEM). As shown in Fig. 1a and b, the structural framework of the porous carbon material was irregular and amorphous. SEM shown the loose porous nature of the material more clearly, suggesting that it may have good pore properties and adsorption properties. Two peaks shown in Raman spectrum (Fig. 1c) were located at $1331 \mathrm{~cm}^{-1}$ (D-band) and $1592 \mathrm{~cm}^{-1}$ (G-band) respectively, where the D-band represented the disorder carbon or defective graphitic structure and the $\mathrm{G}$ band represented the order feature of graphite carbon structure. The $I_{\mathrm{D}} / I_{\mathrm{G}}$ was 1.004 , indicating some defective features in 
a)
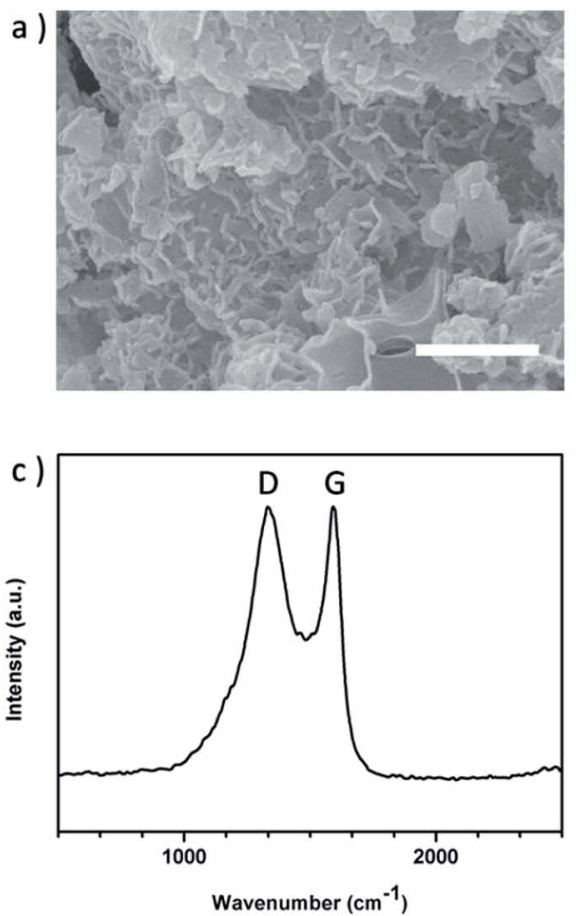

b )
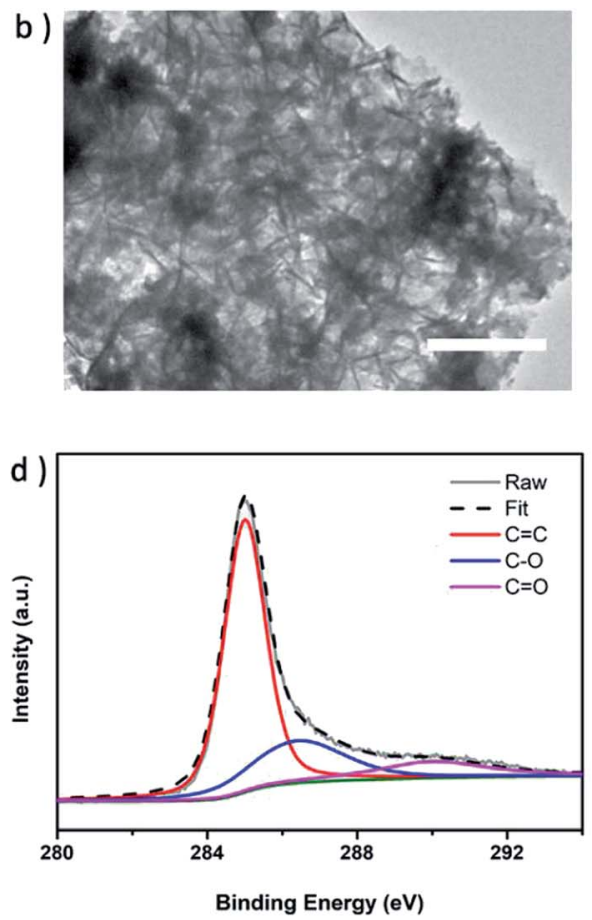

Fig. 1 (a) SEM (scale bar: 500 nm), (b) TEM (scale bar: 500 nm), (c) Raman spectrum, (d) XPS spectra of TS-C.

the porous carbon network structure. ${ }^{18} \mathrm{X}$-ray photoelectron spectroscopy (XPS) revealed mainly carbon content and a small portion of oxygen in porous carbon samples. In the XPS C1s spectrum (Fig. 1d), there were several primary peaks with binding energies of 285.0, 286.5 and $290.1 \mathrm{eV}$, which corresponded to the signals of $\mathrm{C}=\mathrm{C}$ bond, $\mathrm{C}-\mathrm{O}$ bond and $\mathrm{C}=\mathrm{O}$ bond respectively. The reason for the presence of oxygen may be that porous carbon was exposed to the air and the surface is oxidized. ${ }^{19}$

To assess the porous properties of carbon material, the BET specific surface area of the sample was determined by measuring the nitrogen adsorption and desorption curves of the sample under $77 \mathrm{~K}$ after vacuum degassing. As shown in Fig. 2a, the nitrogen adsorption and desorption curve of the porous carbon material was similar to the reversible adsorption curve of type I. A sharp increase at low relative pressure, indicated massive micropores in porous carbon network structure.

After calculation, the specific surface area of the material was $1416 \mathrm{~m}^{2} \mathrm{~g}^{-1}$. The formation of high specific surface area may be attributed to the fact that $\mathrm{KOH}$, as an activator, can etch light elements to generate a large number of pore structures, and the release of $\mathrm{CO}_{2}$, which was generated by the further decomposition of $\mathrm{K}_{2} \mathrm{CO}_{3}$ obtained from the reaction of $6 \mathrm{KOH}+2 \mathrm{C} \rightarrow 2 \mathrm{~K}$ $+3 \mathrm{H}_{2}+2 \mathrm{~K}_{2} \mathrm{CO}_{3}$, promoted the production of more micropores or ultrapores. ${ }^{20}$

With the acceleration of industrialization, large quantities of wastewater are emitted from plastics, textiles, leather, paper, printing, and dye manufacturing plants. It contains a large amount of organic dyes, which is an important factor causing water pollution. Therefore, it was of great significance to explore the adsorption property of porous carbon materials in organic dyes in water system. ${ }^{21,22}$ As shown in Fig. 3, three representative dyes - Congo red (CR), Gentian violet (GV) and Methyl blue (MB) - were selected for the study. Gentian violet is a cationic dye, while Congo red and Methyl blue are anionic dyes. The porous carbon material was placed in aqueous solutions of three

a)
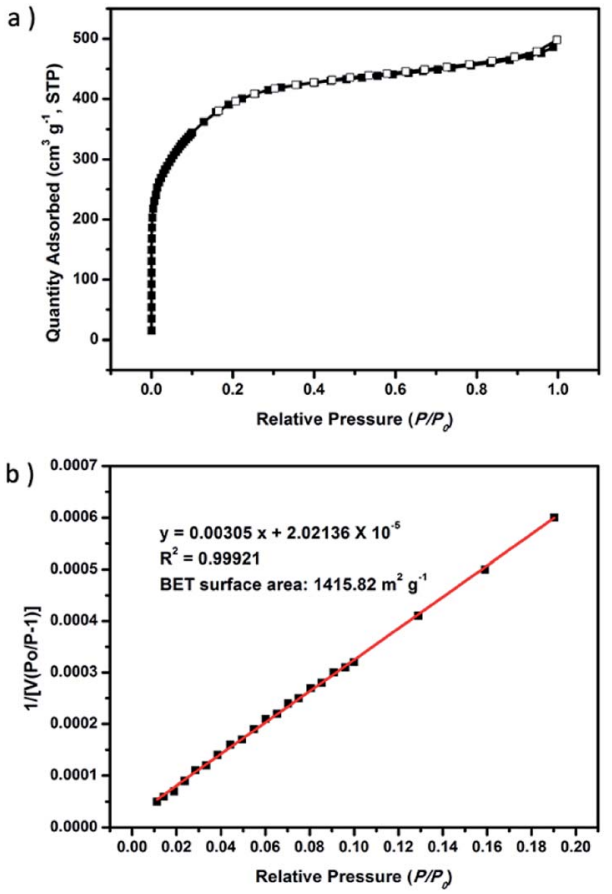

Fig. 2 (a) Nitrogen sorption isotherms at $77 \mathrm{~K}$, (b) Brunauer-EmmettTeller equation. 


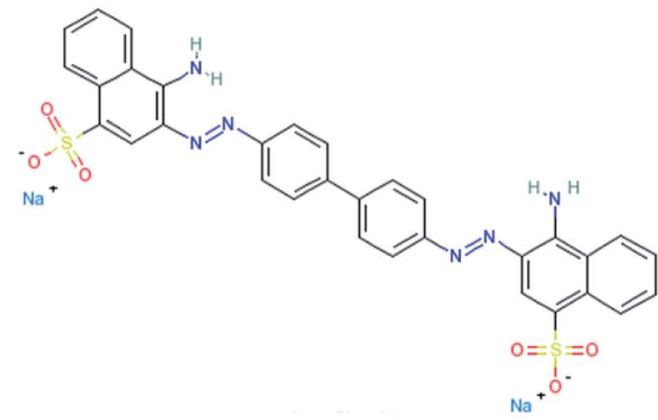

(a)

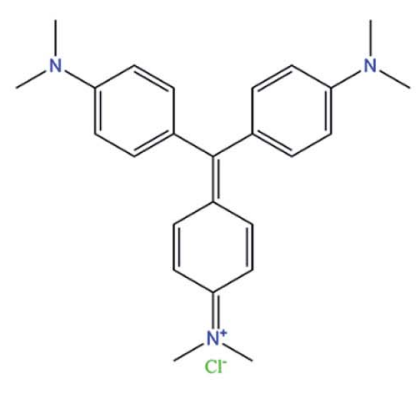

(b)

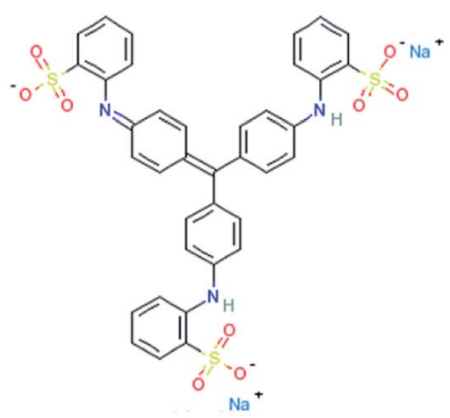

(c)

Fig. 3 Molecular structure of three organic dyes: (a) Congo red, (b) Gentian violet, (c) Methyl blue.

organic dyes, and the supernatant was taken at different time points to measure and obtain UV-vis spectrums (Fig. 4a-c). It can be clearly seen that the ultraviolet absorption of the solution gradually decreased over time until completely disappeared. Then, selecting the peaks at the maximum absorption wavelengths of 497, 590, and $625 \mathrm{~nm}$ for CR, GV and $\mathrm{MB}$, respectively, we obtained the ratio of solution absorbance at different time points and initial solution absorbance. As shown in Fig. $4 \mathrm{~d}-\mathrm{f}$, the decreasing trend of ultraviolet absorption intensity of solution, that was, the decreasing trend of organic dye concentration in aqueous solution. It can also be seen from the vignette in Fig. 4 that the colors of the three organic dye aqueous solutions gradually changed from red, purple and blue to colorless, which visually proved that the porous carbon material adsorbed the organic dyes in the water.

In order to further study the adsorption behavior of porous carbon materials, we conducted an experiment on the adsorption capacity of organic dyes, and used the Langmuir isothermal adsorption model to fit the adsorption data (Fig. 5). The correlation coefficients of the isothermal adsorption curve $\left(R_{\mathrm{L}}^{2}\right)$ were $0.997,0.994$ and 0.991 . It indicated that the fitting results were good and the adsorption process conforms to the assumption of Langmuir isothermal adsorption curve, which was homogeneous chemical adsorption. According to the isothermal adsorption line equation, the maximum adsorption capacity of TS-C on the three organic dyes was calculated as 662, 926, and $179 \mathrm{mg} \mathrm{g}^{-1}$ for $\mathrm{CR}, \mathrm{GV}$ and $\mathrm{MB}$, respectively. Among them, the material had the maximum adsorption on the GV. The reason why the material adsorbed the gentian violet dye higher than the other two anionic dyes may be that the positively charged ions of GV had a better combination with the negatively charged groups in the material. And as shown in Table 1, the carbon material we prepared had a good adsorption capacity of MB compared to most of the reported materials.
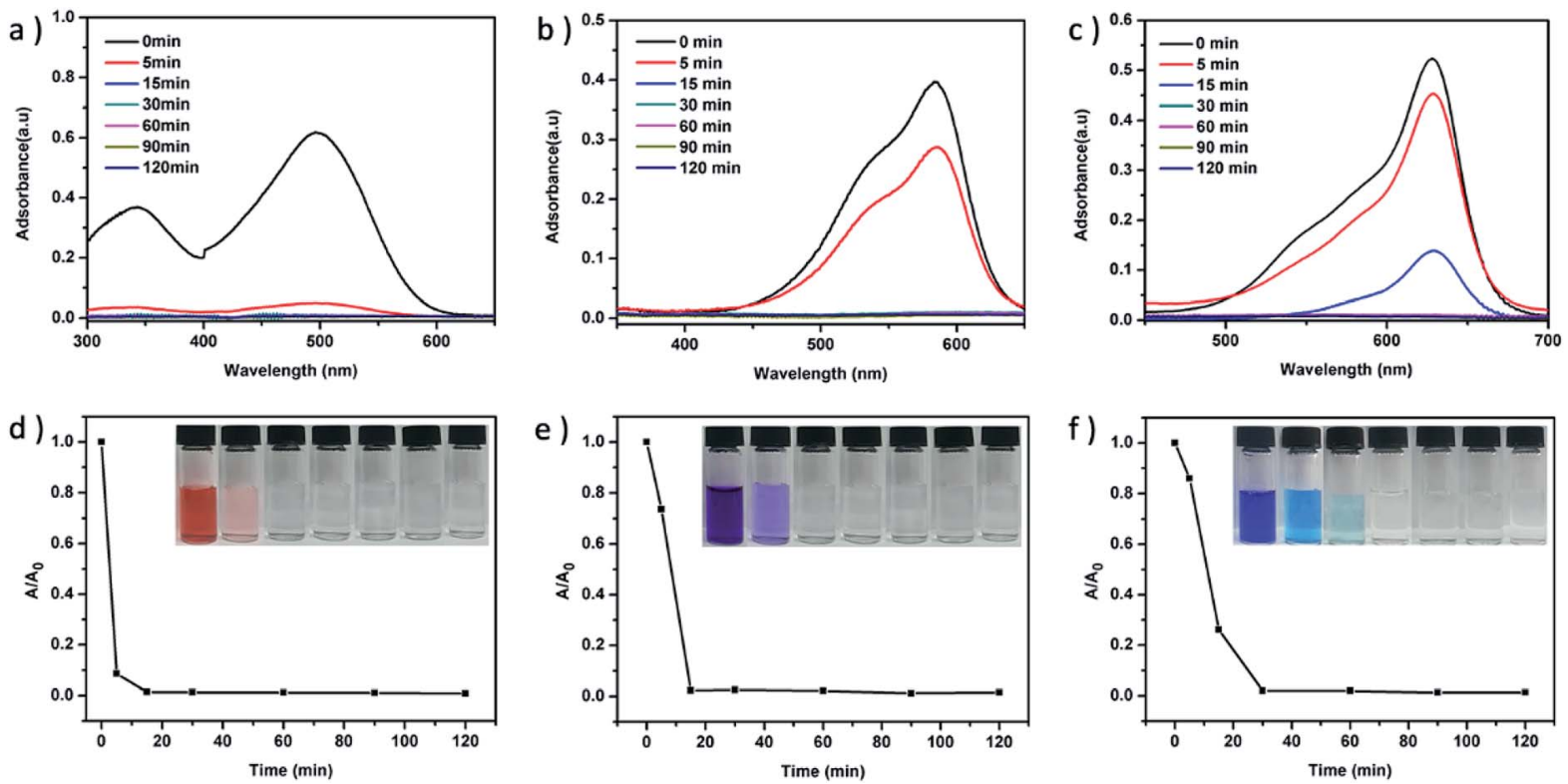

Fig. 4 UV-vis adsorption spectra of the aqueous solution of three organic dyes in the presence of porous carbon at different intervals: (a) Congo red, (b) Gentian violet, (c) Methyl blue; adsorption rates of three organic dyes on porous carbon, the inset shows the corresponding photographs of color change: (d) Congo red, (e) Gentian violet, (f) Methyl blue. 

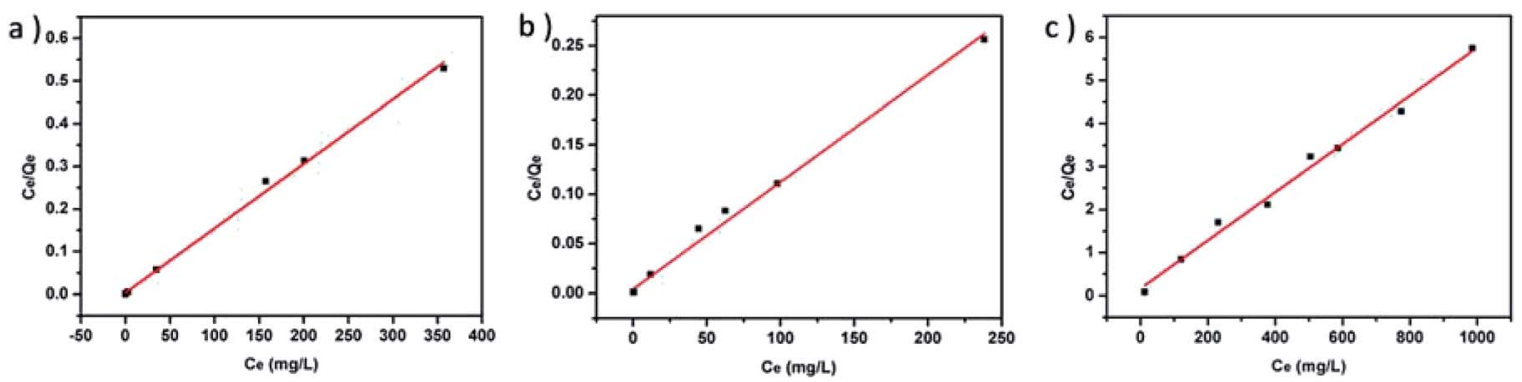

Fig. 5 The adsorption isotherm data of three dyes were fitted using Langmuir isotherm model: (a) Congo red, (b) Gentian violet, (c) Methyl blue.

Table 1 Adsorption capacity and adsorption conditions of MB for various materials recently reported

\begin{tabular}{llcr}
\hline Adsorbent & Temperature $\left({ }^{\circ} \mathrm{C}\right)$ & Adsorbent capacity (mg MB per g) & Reference \\
\hline Activated lignin-chitosan extruded pellets & 20 & 36 & 23 \\
Carbon nanotubes & 25 & 46 & 24 \\
Cu ${ }_{2}$ O-DW & 30 & 53.37 & 25 \\
Fe/SCD-LDH & 30 & 94 & 26 \\
PDOPA-f-LDH & 25 & 132 & 27 \\
TS-C & 25 & 787.40 & Our work \\
THPS & 25 & 12
\end{tabular}

\section{Experimental}

\section{Synthesis of porous carbon}

Tobacco stalk $(1.0 \mathrm{~g})$ was pulverized and transferred to ceramic boat placed into a tube furnace, heating up to $500^{\circ} \mathrm{C}$ at nitrogen gas flow with a rate of $5{ }^{\circ} \mathrm{C} \min ^{-1}$. The reaction was carried at this temperature for $2 \mathrm{~h}$. After cooling to room temperature, $\mathrm{KOH}$ was added to the tobacco stalk, and then heated to $700^{\circ} \mathrm{C}$, and reacted for $2 \mathrm{~h}$ under the same reaction environment. The resultant compound was washed with ultrapure water and centrifuged 2-3 times and then dried with vacuum at $60{ }^{\circ} \mathrm{C}$ for $24 \mathrm{~h}$.

\section{Organic dye adsorption experiment}

Porous carbon material $(5 \mathrm{mg})$ was added into the Congo red aqueous solution with a concentration of $100 \mathrm{mg} \mathrm{L}^{-1}$ and a volume of $4 \mathrm{~mL}$. After magnetic stirring at room temperature, it was removed at different time points ( $0 \mathrm{~min}, 5 \mathrm{~min}, 15 \mathrm{~min}$, $30 \mathrm{~min}, 60 \mathrm{~min}, 90 \mathrm{~min}, 120 \mathrm{~min}$ ). The supernatant was collected to measure the UV-vis absorbance. The residual Congo red concentration was calculated with reference to the UV-vis standard curve of Congo red solution. The adsorption experiment procedure of gentian violet and methyl blue was the same as above.

\section{Experiment on the adsorption capacity of organic dyes}

The initial concentration of $4 \mathrm{~mL}$ confocal red solution was 200 , 300, 400, 500, 600, 700, 800, 1000, 1200, 1400, and $1600 \mathrm{mg} \mathrm{L}^{-1}$. Porous carbon samples $(5 \mathrm{mg})$ were added and magnetized overnight to achieve equilibrium adsorption. After separation by centrifugation $(10000 \mathrm{rpm}, 10 \mathrm{~min})$, the solution was detected by UV-vis adsorption spectra. The data were fitted to the Langmuir isotherm model $\left(C_{\mathrm{e}} / Q_{\mathrm{e}}=C_{\mathrm{e}} / Q_{\mathrm{m}}+1 /\left(K_{\mathrm{L}} Q_{\mathrm{m}}\right)\right)$, where $Q_{\mathrm{e}}\left(\mathrm{mg} \mathrm{g}^{-1}\right)$ is the equilibrium adsorption, $C_{\mathrm{e}}\left(\mathrm{mg} \mathrm{L}^{-1}\right)$ is the equilibrium concentration of Congo red dye, $Q_{\mathrm{m}}\left(\mathrm{mg} \mathrm{g}^{-1}\right)$ is the maximum adsorption, and $K_{\mathrm{L}}$ is the Langmuir constant. The adsorption capacity of gentian violet and methyl blue was tested as above.

\section{Conclusions}

In summary, we have prepared porous carbon material TS-C with $\mathrm{KOH}$ as activator and agricultural residue tobacco stalk as precursor by high temperature carbonization. The obtained porous carbon materials have high specific surface area and good adsorption performance of organic dyes. The application of porous carbon material prepared by tobacco stalk in the adsorbent undoubtedly provides a new idea for environmental protection and waste utilization.

\section{Conflicts of interest}

There are no conflicts to declare.

\section{Acknowledgements}

This work is supported by the National Natural Science Foundation of China (21875079), and the Fine Branch Innovation Major Projects of Hubei Tobacco (Group) Co., Ltd. China (2018A028). We also thank the Analytical and Testing Center of Huazhong University of Science and Technology for related analysis. 


\section{References}

1 V. Strezov, E. Popovic, R. V. Filkoski, P. Shah and T. Evans, Energy Fuels, 2012, 26, 5930-5935.

2 K. Cong, F. Han, Y. Zhang and Q. Li, Fuel, 2019, 237, 126-132.

3 J. Liu, G. Ma, T. Chen, Y. Hou, S. Yang, K. Q. Zhang and J. Yang, Appl. Microbiol. Biotechnol., 2015, 99, 3775-3785.

4 B. Yan, S. Zhang, W. Chen and Q. Cai, J. Anal. Appl. Pyrolysis, 2018, 136, 248-254.

5 G.-N. Guo, B. Cai, R. Li, X. Pan, M. Wei and C. Zhang, Biomass Convers. Biorefin., 2019, DOI: 10.1007/s13399-019-00478-2.

6 L. Li, R. Wang, Z. Jiang, W. Li, G. Liu and C. Chen, Environ. Sci. Pollut. Res. Int., 2019, 26, 14250-14258.

7 G. Tuzzin, M. Godinho, A. Dettmer and A. J. Zattera, Carbohydr. Polym., 2016, 148, 69-77.

8 N. Hiyoshi, O. Sato, A. Yamaguchi, C. V. Rode and M. Shirai, Green Chem., 2012, 14, 633.

9 J. Wang, T. Zhang, M. Li, Y. Yang, P. Lu, P. Ning and Q. Wang, RSC Adv., 2018, 8, 22694-22709.

10 Y. Zhai, Y. Dou, D. Zhao, P. F. Fulvio, R. T. Mayes and S. Dai, Adv. Mater., 2011, 23, 4828-4850.

11 K. Zhang, B. Tieke, F. Vilela and P. J. Skabara, Macromol. Rapid Commun., 2011, 32, 825-830.

12 C. Zhang, P.-C. Zhu, L. Tan, J.-M. Liu, B. Tan, X.-L. Yang and H.-B. Xu, Macromolecules, 2015, 48, 8509-8514.

13 Q.-M. Zhang, Z. Wang, G. Cheng, H. Ma, Q.-P. Zhang, F.-X. Wan, B. Tan and C. Zhang, RSC Adv., 2018, 8, 3339833402.

14 Q. M. Zhang, T. L. Zhai, Z. Wang, G. Cheng, H. Ma, Q. P. Zhang, Y. H. Zhao, B. Tan and C. Zhang, Adv. Mater. Interfaces, 2019, 6, 1900249.
15 C. Xuan, Z. Peng, J. Wang, W. Lei, K. Xia, Z. Wu, W. Xiao and D. Wang, Chin. Chem. Lett., 2017, 28, 2227-2230.

16 M. Sevilla, G. A. Ferrero and A. B. Fuertes, Chem. Mater., 2017, 29, 6900-6907.

17 Y. Wang, T. Liu, X. Lin, H. Chen, S. Chen, Z. Jiang, Y. Chen, J. Liu, J. Huang and M. Liu, ACS Sustainable Chem. Eng., 2018, 6, 13932-13939.

18 X. Liu, Y. Zhou, W. Zhou, L. Li, S. Huang and S. Chen, Nanoscale, 2015, 7, 6136-6142.

19 Y. Hu, X. Tong, H. Zhuo, L. Zhong and X. Peng, ACS Sustainable Chem. Eng., 2017, 5, 8663-8674.

20 D. Li, Q. Wang, Y. Yao, F. Wu, Y. Yu and C. Zhang, ACS Appl. Mater. Interfaces, 2018, 10, 32058-32066.

21 Y. Hao, Z. Wang, Z. Wang and Y. He, Ecotoxicol. Environ. Saf., 2019, 168, 298-303.

22 X. Wen, H. Liu, L. Zhang, J. Zhang, C. Fu, X. Shi, X. Chen, E. Mijowska, M. J. Chen and D. Y. Wang, Bioresour. Technol., 2019, 272, 92-98.

23 A. B. Albadarin, M. N. Collins, M. Naushad, S. Shirazian, G. Walker and C. Mangwandi, Chem. Eng. J., 2017, 307, 264-272.

24 Y. Yao, F. Xu, M. Chen, Z. Xu and Z. Zhu, Bioresour. Technol., 2010, 101, 3040-3046.

25 X. Tao, Y. Wu and H. Sha, Water, Air, Soil Pollut., 2018, 229. 26 W. Hu, X. Wu, F. Jiao, W. Yang and Y. Zhou, Desalin. Water Treat., 2016, 57, 25830-25841.

27 J. Zhao, Q. Huang, M. Liu, Y. Dai, J. Chen, H. Huang, Y. Wen, X. Zhu, X. Zhang and Y. Wei, J. Colloid Interface Sci., 2017, 505, 168-177. 